\title{
Registration Center Telecom Address
}

National Cancer Institute

\section{Source}

National Cancer Institute. Registration Center Telecom Address. NCI Thesaurus. Code C94282.

A sequence of digits or characters used to identify a particular telephone, fax, or email of a registration center. 